\title{
Time judgments of musical endings: Effects of expectancies on the "filled interval effect"
}

\author{
MARILYN BOLTZ \\ Haverford College, Haverford, Pennsylvania
}

\begin{abstract}
In two experiments, the influence of musical endings on duration judgments was examined. In Experiment 1, subjects were asked to compare the duration of paired folk tunes that could vary in their degree of resolution. Results showed that melodies ending on the leading tone interval were underestimated relative to songs ending on the conventional tonic. Experiment 2 further revealed that time estimates were influenced by a melody's preceding temporal accent structure. Melodies ending earlier than expected were judged significantly shorter, particularly when the tune ended on the leading-tone interval. Conversely, tonic endings were judged substantially longer when they appeared to occur "too late" in time. Results are discussed in terms of a contrast model which emphasizes the roley oxpectancies on duration judgments.
\end{abstract}

We've all been to the movies and noticed that some films seem longer or shorter than others, even though they're all approximately an hour and a half in length. Similarly, one song on the radio may seem longer than another, even though both are actually the same duration. These phenomena illustrate a primary emphasis of current time research known as the filled interval effect. That is, two equivalent intervals of time are not always judged to have the same duration but, instead, one may seem longer or shorter than another depending on the type of information "filling" the time spans themselves (see Allan, 1979; Fraisse, 1984 for reviews). The purpose of the present set of experiments was to investigate this effect in the context of a natural event-namely, musicand to consider how time estimates may be influenced by manipulations of musical endings.

\section{Contemporary Models of Time Estimation}

The most influential models of time estimation that address the filled interval effect share, to a certain extent, some basic underlying assumptions. Perhaps the most central of these is that time judgments depend on the amount of cognitive activity required to process a given stimulus. Stimuli that are more complex will seem longer because they require greater processing than do less complex stimuli. Yet, although all models share this assumption, they vary in terms of what particular cognitive activity is used as the referent for duration judgments. In general, these models can be categorized into one of three types.

\footnotetext{
This research was conducted while the author was a visiting assistant professor at Ohio State University. The author thanks Mari R. Jones for the use of her laboratory and also Jean Spizzirri for assistance with the manuscript's preparation. Requests for reprints should be addressed to Marilyn Boltz, Department of Psychology, Haverford College, Haverford, PA 19041. Bitnet address: M_BOLTZ@HVRFORD.
}

The earliest, and probably the most well-known model, is the "storage size hypothesis" proposed by Ornstein (1969). According to this view, two equal time intervals may seem to have different durations because one is more complex and therefore requires more storage space in memory. In support, Ornstein (1969) conducted experiments in which complexity was defined in terms of the number of angles in abstract drawings or randomized (vs. blocked) presentations of auditory and visual sequences. Experienced duration did lengthen with greater complexity - a finding later corroborated by others (Hogan, 1975; Schiffman \& Bobko, 1974).

A second class of models assumes that judged duration depends on the amount of attentional effort required by a given stimulus (Thomas \& Weaver, 1975; Underwood \& Swain, 1973). That is, complex stimuli seem longer because they require greater selective attention. Studies supporting this idea have found that intervals containing a higher degree of background noise are overestimated in duration, even though less information in these intervals is retained in memory (Underwood \& Swain, 1973).

The third type of model, proposed by Block (1979, 1985 , in press), is known as the "cognitive change hypothesis." The basic assumption of this approach is that judged duration increases with the number of contextual changes within the organism (e.g., changes in processing strategy) or the environmental situation (e.g., changes in task demands, stimulus structure, etc.). This model also has accrued empirical support as exemplified by a study where it was found that duration estimates increased when subjects performed multiple tasks (vs. a single task) during a given time interval (Block \& Reed, 1978).

In sum, contemporary models claim that judged duration depends on the amount of memory storage, attentional effort, or cognitive change. Although each model defines complexity in a somewhat different fashion, all assume 
that more complex stimuli will seem longer because they require greater processing activity.

These models have generated a substantial amount of research and have provided theoretical frameworks from which to investigate the filled interval effect. Nevertheless, the current literature is not without difficulties. The most serious problem is that the empirical research is characterized by divergent and conflicting results. An interval defined as more complex is sometimes judged longer, but on other occasions shorter, than a less complex interval. For example, conflicting results have been observed with the variables of stimulus familiarity (cf. Avant \& Lyman, 1975; Devane, 1974), stimulus arrangement (cf. Poynter, 1983; Schiffman \& Bobko, 1974), and task difficulty (cf. Burnside, 1971; Underwood \& Swain, 1973).

Jones and Boltz (1989) have suggested that the divergences among these results stem from multiple sources, including methodological differences across various studies as well as problems associated with certain underlying assumptions in the models themselves. One issue of particular concern involves the idea that time judgments depend on the processing of strictly nontemporal information. Ornstein's (1969) model, for example, assumes that experienced duration is inferred from the number and arrangement of stimulus items, whereas Block's (1988) approach assumes the mediation of stimulus and observer change. And although nontemporal information clearly does exert a significant impact on duration estimates, it is also possible that the temporal patterning of such information may be of equal importance. People may attend differently to events that unfold over a predictable as opposed to an unpredictable time course and this in turn may differentially influence time estimation activities.

\section{An Expectancy/Contrast $/$ Model of Judged Duration}

An alternative model of judged duration, initially suggested by Boltz (1985) and later elaborated by Jones and Boltz (1989), has in fact adopted this notion as its fundamental thesis. That is, events within the natural environment are assumed to vary along a continuum of structural coherence and predictability. Highly coherent events are those that contain a characteristic time span marked by a nonarbitrary beginning and end, and whose various levels of structure all mesh and interrelate in a very lawful, predictable fashion. Examples of such events include speech utterances, melodies, the gait pattern of a pedestrian, or even a lecture whose content is highly organized and flows without interruption. Less coherent events, on the other hand, typically unfold over more arbitrary time spans and lack predictability in their structural arrangement. These might be exemplified by a list of nonsense or unrelated words, an unorganized lecture that is frequently interrupted, or the simultaneous chatter of a cocktail party. The basic premise is that people attend differently to events with high or low structural coherence and that this in turn differentially affects time estimates.
Highly coherent events are assumed to afford futureoriented attending. Given their predictability, a perceiver is able to extrapolate the event's preceding context and generate expectancies about upcoming structural relationships, including how and when the event will end. In these situations, Jones and Boltz (1989) have proposed, duration estimates are determined by whether an event's ending confirms or violates one's expectancies. In terms of the filled interval effect, if two events have equivalent total durations and both end "when" expected, then people will correctly judge them to be the "same." However, if one event violates an expectancy by seeming to end later than anticipated, it will be incorrectly judged as longer. Similarly, events appearing to end "too early" will be judged as relatively short. According to this view, then, duration estimates depend on temporal contrast, where contrast involves a temporal disparity between an event's actual and expected ending.

Other events in the natural environment display less structural coherence and predictability. They typically lack a naturally marked beginning and end, and their lack of internal organization forces us to cope with seemingly unrelated circumstances. Hence, these events do not allow future-oriented attending. Instead, they enforce analytical attending, because people must attend to relationships among adjacent items within the time span. In order to achieve organization and meaning, people must rely on cognitive strategies that supply missing relationships to the event's structure. And the attempt to organize and integrate these events may be reflected in duration estimates. That is, a person may try to connect the unrelated elements of a time span through grouping, chunking, or other cognitive strategies. When asked to judge relative duration, a person may then count the number of individual items, chunks, (à la Ornstein, 1969), or changes (à la Block, 1985) to infer duration differences.

In sum, the contrast model assumes that duration estimates are determined by the structure of events to be judged. Events that allow future-oriented attending, through structural predictability, are judged in terms of whether their endings confirm or violate one's expectancies. Conversely, less coherent events reflect the use of mnemonic strategies that aid in the organization and integration of the event. Here, people rely on whatever information is available to infer duration estimates, and this may include counting the number of chunks or changes within a time span.

Empirical support for this view comes from a set of studies by Jones and Boltz (1989). In these experiments, subjects were asked to compare the relative durations of folk tunes that varied in their temporal accent structures. These melodies were designed to promote future-oriented attending in that temporal (agogic) accents always maintained a lawful relationship with pitch intervals marking the end of melodic phrases (i.e., tonic triad members), and, at least initially, these accents always recurred after an invariant number of beats. However, in some versions of the melodies, the timing of the final accent phrase was 
manipulated such that it contained a fewer or greater number of beats than that of preceding phrases. Results indicated that melodies whose end occurred relatively "too early" or "too late" in time produced under- and overestimations, respectively, even though all melodies were equivalent in total duration and total number of beats. Thus, duration estimates of coherent events do seem to depend, at least in part, on expectancies extrapolated from the events' preceding structural relationships.

\section{Rationale of the Present Experiments}

The purpose in the present experiments was to investigate the effects of other types of expectancies on duration judgments-namely, ones involving the very last notes of musical compositions. Jones and Boltz (1989) have examined how a melody's overall rhythmic context affects expectancies about when the last note of a tune should occur, but other time distortions may emerge if properties of the last note itself are varied. There is evidence that most Western music does in fact display a nonarbitrary ending marked by certain structural characteristics. That is, most compositions end on a progression from leading tone to tonic, where tonic refers to the underlying key in which a melody is written. For example, the melody shown at the top of Figure 1 is based on the $\mathrm{C}$ major diatonic scale and thus ends on the note $\mathrm{C}$. But since the tonic can recur throughout the course of a melody, a musician will mark melodic completion in two additional ways: First, the leading tone, which precedes the tonic in the underlying diatonic scale ( $B$ in the $C$ major scale), acts as the penultimate note of a tune and thereby creates an expectancy for an immediately ensuing tonic. Second, the duration of the final tonic is prolonged relative to the durations of all preceding notes. The conventional use of these structural markers is known as resolution, and it is tacitly understood to communicate melodic completion (Berry, 1976; Meyer, 1956; Piston, 1978). A similar phenomenon has been observed in speech: speakers often begin and end declarative statements with the same pitch frequency (Cooper \& Sorensen, 1977) and prolong the duration of the final word (Cooper, Paccia, \& LaPointe, 1978; Scott, 1982).

Given that resolution is typical of most Western music, it is reasonable to suppose that listeners have tacitly learned this convention and expect to hear a final prolonged tonic at the end of a song. If so, then melodic

a. INVARIANT

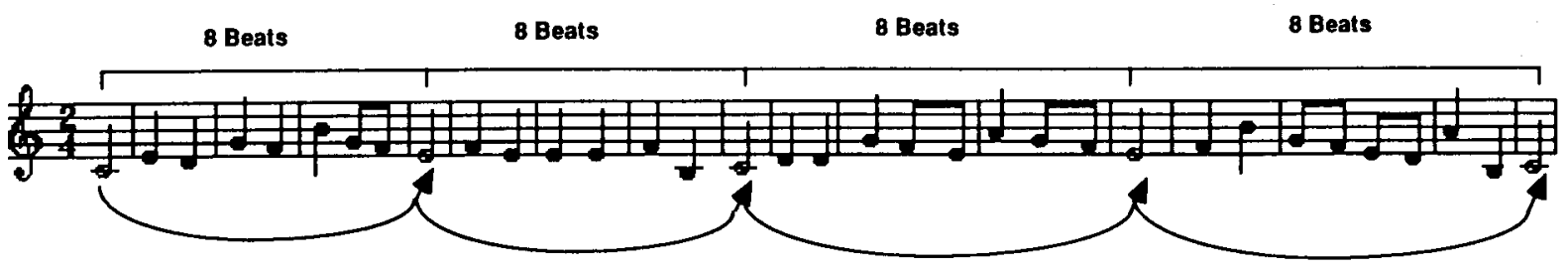

b. ACCENT EARLY

EXPECTANCIES

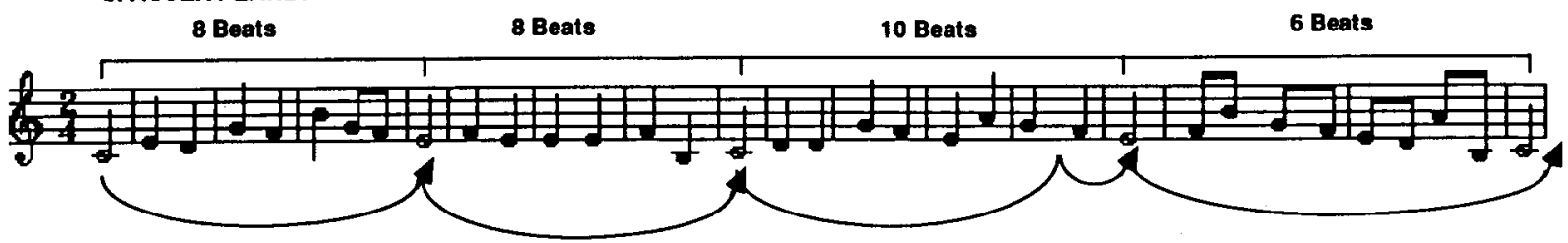

c. ACCENT LATE

EXPECTANCIES

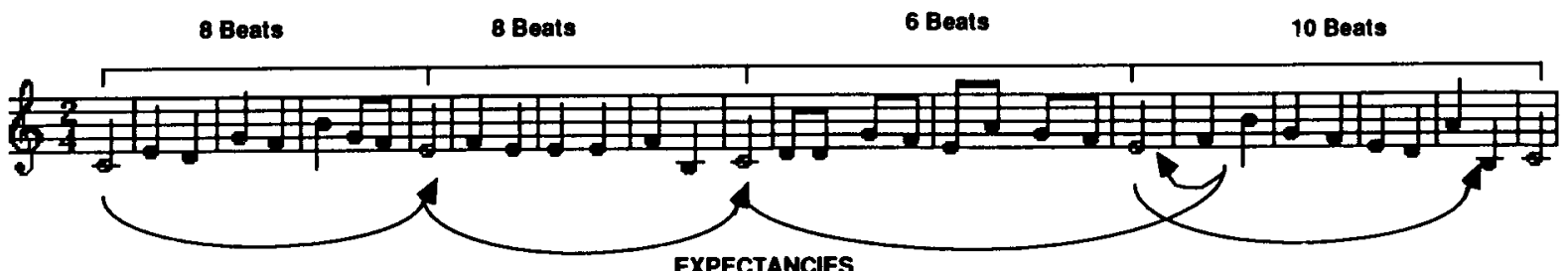

Figure 1. An exemplar set of melodies used as experimental stimuli. The invariant melody (a) depicted at the top illustrates the concept of resolution in that it ends on the progression from leading tone to tonic (B-C). A lack of resolution, as defined in Experiments 1 and 2 , arises from the reversed temporal ordering of these two pitch intervals (C-B). This melody displays two other structural characteristics commonly found in Western music: the temporal accentuation $(d)$ of tonic triad members (here the tonic, $C$, and the mediant, E) and a recurrent periodicity of these coupled accents after an invariant number of beats (i.e., 8 beats). The temporal predictability of these coupled accents allows a listener to generate expectancies (shown as arrows) about the "what" and "when" of upcoming accents, which here are reliably confirmed. The accent early melody (b) also contains coupled accents, but the timing of accents in the third and fourth periods has been manipulated such that the melody's ending occurs earlier than expected. Similarly, the temporal regularity of accents in the accent late melody (c) has been varied such that the melodic ending occurs later than expected. 
endings marked by other pitch intervals should appear both incomplete and inappropriate to a melody's tonality. The previous literature indicates that this is the case. In a study by Boltz (in press), subjects were asked to judge the degree of "completeness" for a set of folk tunes that varied in their tonal endings. Results showed that melodies ending on the progression from leading tone to tonic were judged the most complete, more so than songs ending with the submediant-to-tonic or the tonic-to-dominant progressions. Conversely, melodies ending on the leading tone seemed to be the most incomplete because the listeners were left "hanging" without a final return to tonicity. Cuddy, Cohen, and Miller (1979) have reported that melody recognition is more accurate for tunes ending on the tonic than those ending on other diatonic scale intervals. Lastly, Sundberg, Friberg, and Frydén (1987) have demonstrated a sensitivity to the temporal properties of resolution in that listeners prefer songs ending with prolonged tonal durations.

Experiment 1 was designed to determine whether violations of musical resolution also exert an impact on relative duration judgments. Subjects were asked to compare the relative durations of a pair of folk tunes that could vary in their tonal endings. On some trials, both melodies were resolved in that each ended on the conventional progression from leading tone to tonic, or both were unresolved in that they ended on the progression from tonic to leading tone. The trials of most interest, however, were those in which one song of a pair was resolved and the other was unresolved. Assuming that the leading tone creates an expectancy for an immediately ensuing tonic, unresolved melodies may seem shorter than their resolved counterparts. These melodies seem as if they should continue on to the tonic, and since they don't, they leave a listener "hanging" with an end that occurs "too early" in time. Thus, relative to resolved melodies, unresolved melodies should produce underestimations of judged duration while overestimations should arise whenever a resolved tune is judged relative to an unresolved one.

In addition to the manipulation of tonal ending, the temporal ending of melodies was also varied. On some trials, melodies within a pair both ended on a final note that was significantly longer than any preceding note, thus conforming to the temporal characteristics of resolution. On other trials, however, each melody of a pair ended on a note (i.e., an eighth or a quarter note) that was significantly shorter than expected according to the convention of resolution. It was thought that the latter conditions might enhance any effects of the leading-tone ending, since these melodies were now both temporally and tonally foreshortened in time. Thus, the degree of underestimation associated with a leading-tone ending might substantially increase.

Although the contrast model predicts unresolved melodies to yield underestimations of relative duration, more traditional models of time estimation generate a very different pattern of results. Both the storage size (Ornstein, 1969) and the attentional effort models (Underwood
\& Swain, 1973) would predict a uniform set of same judgments for all trials since variations of the final note should have no effect on either selective attention or the number of chunks in memory. The cognitive change hypothesis (Block, 1985, in press), on the other hand, might predict that any deviations from an end-lengthened tonic should increase stimulus complexity and, thus, experienced duration. In this view, then, unresolved melodies should be overestimated relative to resolved ones. To contrast these various sets of predictions, the following experiment was conducted.

\section{EXPERIMENT 1}

\section{Method}

Design and Subjects. The design was a $3 \times 3 \times 2 \times 2 \times 2$ mixed factorial. Melodies within a pair always shared one of three folk tunes, one of three temporal endings (isochronous-isochronous; long-long; short-short) and could share a common (tonic-tonic; leading tone-leading tone) or different (tonic-leading tone; leading tone-tonic) tonal ending that varied in degree of resolution. Counterbalance order (I, II) was the single between-subjects factor.

Twenty-four subjects from an introductory psychology course at Ohio State University participated in the experiment in return for course credit. Each had had at least 4 years of musical experience within the past 6 years and was currently playing a musical instrument. Twelve subjects were randomly assigned to one of two counterbalance orders.

Stimulus materials. Excerpts of three folk tunes were selected from a music composition book. The original titles of these songs were: "There's Music in the Air,"' by George Root; "Softly Now the Light of Day," by George Doane; and "I Don't Want to Play in Your Yard," by H. W. Petrie. To highlight the melody's tonality, each excerpt was modified such that (1) the first and last notes were always the tonic, and (2) the rare tritone interval, reported to be the single best indicant of a melody's key (Browne, 1981; Butler \& Brown, 1984), appeared in both the first and final accent periods of each song.

These melodies represent a somewhat diverse range of music in that they differ in total number of notes (range $=34$ to 37 notes), meter $(2 / 4,4 / 4)$, and musical key (C, G, F major). All, however, contain an invariant number of beats between agogic accents (i.e., 8 beats); accents that correspond to members of the tonic triad chord (i.e., tonic, mediant); and the same stimulus-onset asynchrony value for quarter notes $(J=300 \mathrm{msec}$ ), eighth notes $(\delta=150 \mathrm{msec})$ and half notes $(\delta=600 \mathrm{msec}$ ).

The tonal ending of each of these three tunes was then varied such that the melody in its resolved form displayed an ending with a progression from leading tone to tonic. In the melody's unresolved form, the temporal order of the last two notes was reversed such that the melody ended on the interval from tonic to leading tone.

The temporal endings of this set of 6 melodies were also manipulated. In the isochronous condition, the duration of the final note was equivalent to that of the melody's beat unit-namely, a quarter note (i.e., note on time $=200 \mathrm{msec}$ ). Conversely, the final note duration in the short condition was equal to that of an eighth note (i.e., $100 \mathrm{msec}$ ), whereas in the long condition, it was $67 \%$ longer than the duration of a half note (i.e., $800 \mathrm{msec}$ ).

Melodies were then paired for pattern presentation such that the final pitch intervals of the two tunes were either the same (i.e., tonic-tonic; leading tone-leading tone) or different (tonic-leading tone; leading tone-tonic). All pattern pairs, however, always contained the same song, the same total duration, and the same temporal ending (i.e., short-short; long-long; isochronousisochronous). A factorial crossing of the four types of tonal ending 
with the three types of temporal ending and three songs resulted in a total of 36 experimental trials. Trials were randomized into two blocks of 36 trials, yielding two presentations of each melody for a given subject.

Apparatus. All melodies consisted of square wave tones generated by a Wavetek Model 159 waveform generator that was controlled by a PDP-11 microcomputer. In generation, a custom-built envelope generator imposed 10 -msec rise and fall times on all tones. Tonal frequencies, in hertz, were taken from the equally tempered scale of International Pitch $(\mathrm{A4}=440 \mathrm{~Hz})$ and were subjectively equated for loudness in the actual experimental context according to the reports of two judges. Sequences of tones, programmed by the microcomputer, were recorded on a Nakamichi LX-3 cassette recorder. During experimental sessions, blocks of prerecorded melodies were presented on a Tandberg Model TCD 310MK II cassette player through AKG K 400 headphones at a comfortable listening level.

Procedure. The subjects were told that time judgments would be requested and that they must therefore remove their watches. Recorded instructions informed the subjects of pattern presentation details and task requirements. On each trial, a 1-sec warning tone $(5000 \mathrm{~Hz})$ preceded an initial melody by $2 \mathrm{sec}$. Two seconds later, a second melody was presented. During a 7-sec response interval, the subjects were then asked to judge whether the total duration of the second melody was the same as, longer than, or shorter than the first. Judgments were indicated on a 7-point response scale, with $1=$ very short, $4=$ same duration, and $7=$ very long. After receiving four practice trials, the subjects were then presented with 2 blocks of 36 experimental trials with a brief rest in between. The entire experimental session was approximately $45 \mathrm{~min}$ long.

\section{Results}

Table 1 depicts mean judged duration as a function of tonal and temporal ending pairs. Means are collapsed over song and counterbalance order since both variables contributed negligible effects $(F<1.00)$.

Results indicate that duration judgments varied significantly as a function of whether melodic pairs contained a same or different tonal ending $\left[F(3,66)=23.59, M S_{\mathrm{e}}\right.$ $=0.096, p<.0001]$. When both melodies of a pair contained the same tonal ending, they were correctly judged as equivalent in total duration. However, melodies ending on the leading tone seemed significantly shorter relative to ones ending on the tonic. Conversely, melodies ending on the tonic were overestimated relative to ones ending on the leading tone (Tukey post hocs $<.01$ ). The average absolute differences from a same judgment (i.e.,
4) were 0.32 and 0.43 , respectively, which represent $11 \%$ and $14 \%$ shifts on the rating scale.

The overall ANOVA also revealed that the relative magnitudes of under- and overestimations were the same across all conditions of temporal ending. There was no main effect of the final note's duration nor was there an interaction with tonal ending.

\section{Discussion}

The results of Experiment 1 support predictions of the contrast model in that duration estimates do seem to be influenced by expectancies about musical resolution. Melodies ending on the leading-tone interval were underestimated in relative duration, suggesting that listeners are attuned to the leading tone's role in signaling an immediate return to tonicity. Thus, when the expected final tonic was missing, the melody seemed to have ended too early in time and to be "short" in relative duration. Conversely, melodies ending on the leading tone to tonic progression unfolded to their expected ending point and, in comparison, seemed relatively longer.

Although these findings suggest that listeners are sensitive to the pitch markers of musical completion, results indicate that the temporal characteristics of resolution exerted no effects on duration judgments. This raises two possibilities. First, the prolongation of the final note's duration may simply be less important in marking musical completion than the temporal ordering of pitch information. Alternatively, the present design may have encouraged a greater reliance on pitch information, since both melodies in a pair always shared the same temporal ending but could vary in their final pitch intervals. Thus, subjects may have ignored the temporal variation and focused exclusively on differences in tonal ending. Additional research is needed to clarify these issues and, in particular, to determine the relative weighting of pitch and time information in the communication of musical completion.

Overall, however, the present set of results present difficulties for many contemporary theories of time estimation. For example, the storage size (Ornstein, 1969) and attentional effort (Underwood \& Swain, 1973) models both predicted a generalized pattern of same judgments,

Table 1

Mean Judged Duration (with Standard Deviations) in Experiment 1 as a Function of the Tonal and Temporal Ending of the First and Second Melody Within a Pair

\begin{tabular}{|c|c|c|c|c|c|c|c|}
\hline \multirow[b]{3}{*}{ Tonal Ending Pairs } & \multicolumn{6}{|c|}{ Temporal Ending Pairs } & \multirow[b]{3}{*}{ Mean } \\
\hline & \multicolumn{2}{|c|}{ Isochronous } & \multicolumn{2}{|c|}{ Short } & \multicolumn{2}{|c|}{ Long } & \\
\hline & $M$ & $S D$ & $M$ & $S D$ & $M$ & $S D$ & \\
\hline Tonic-tonic & 3.98 & .20 & 4.02 & .25 & 4.05 & .22 & 4.02 \\
\hline Leading tone-leading tone & 4.05 & .19 & 4.02 & .22 & 4.05 & .24 & 4.04 \\
\hline Tonic-leading tone & 3.67 & .28 & 3.65 & .30 & 3.72 & .31 & 3.68 \\
\hline Leading tone-tonic & 4.42 & .31 & 4.40 & .33 & 4.48 & .32 & 4.43 \\
\hline Means & 4.03 & & 4.02 & & 4.08 & & \\
\hline
\end{tabular}

Note-A value of 4.00 indicates equivalent judged duration; values $<4.00$ indicate that the second melody was judged shorter; and values $>4.00$ indicate that the second melody seemed longer. 
since manipulations of the last note should exert no impact on either memory-code size or selective attention. And yet this was not the case; both over- and underestimations were observed. Similarly, the cognitive change hypothesis encounters problems in that pitch changes within melodic pairs did not uniformly yield overestimations of relative duration. Instead, time judgments depended on the type of change and the particular communicative function that a given pitch relationship typically expresses in Western music.

\section{EXPERIMENT 2}

Although listeners apparently rely on certain structural properties of a final note to discern musical completion, there is other evidence suggesting that listeners are able to anticipate in advance when the last note will occur. Witness, for example, the smooth exchange of lead instruments during a concert performance or an audience's applause that often coincides with the last note of a composition. These phenomena suggest that listeners use certain structural properties not only to attentionally track a melody over time, but to also generate expectancies about upcoming structural relationships that have not yet occurred. What type of information affords this degree of predictability such that one can anticipate precisely when in time an end will occur?

Perhaps the primary vehicle of anticipatory attending is the overall rhythmic structure of events as related to the arrangement of pitch intervals over time. Rhythm refers to the pattern of durational changes as governed by the constraints of meter. For example, the melody shown in Figure 1a is written in a $2 / 4$ meter, indicating that a quarter note $(i)$ receives one beat of time and that there are 2 beats per measure. Within this metric framework, however, a particular rhythm can arise from the shortening or lengthening of tonal durations relative to the referent beat. The half notes $(d)$ shown in Figure 1a, for example, receive two beats of time because their durations are twice those of quarter notes, while eighth notes ( $)$ ) receive half beats because their durations are half those of the beat unit.

One psychological consequence of rhythm is that lengthened notes tend to capture a listener's attention and provide points of temporal accentuation within a melody. Since temporal accents tend to coincide with members of the tonic triad chord (i.e., the mediant, dominant, and tonic) at phrase ending points (Berry, 1976; Cone, 1968; Piston, 1978), rhythm can therefore serve to highlight a melody's underlying tonality (Kramer, 1981; Meyer, 1956; Todd, 1985). In addition, others have suggested that rhythm affords a more dynamic role of guiding attending activities over time (Jones, 1976, 1982; Jones, Boltz, \& Kidd, 1982). That is, temporal accents not only mark melodic phrase endings but also tend to recur after an invariant number of beats. This in turn allows a listener to extrapolate the melody's preceding accent structure to generate expectancies about both the "what" and the "when" of upcoming accents. As is shown in Figure 1a, this includes expectancies about the last note, since it too recurs with the same periodicity (here, 8 beats) as preceding accents and corresponds to the tonic itself.

The purpose of Experiment 2 was to determine whether violations of rhythmic structure may enhance any time distortions arising from manipulations of tonal ending. As in Experiment 1, musically sophisticated listeners were asked to compare the relative durations of paired folk tunes that could vary in their final pitch relationships. In addition, temporal accent structure was manipulated such that on some trials, the second melody relative to the first one in a pair, could appear to end either "on time," or earlier or later than expected given preceding accent periodicities. These manipulations, identical to those used by Jones and Boltz (1989), could assume one of three types. As shown in Figure 1a, some accent patterns were invariant in that agogic accents recurred with a fixed periodicity (i.e., 8 beats) and coincided with tonic triad members marking melodic phrase endings. Thus, the accent structure of this type of melody can be used to reliably anticipate when the end will occur. But next consider the melody labelled accent early in Figure 1. This tune contains the same notes and same total number of beats as its invariant counterpart, as well as agogic accents that coincide with tonic triad members. However, notice that the number of beats between accents has been violated such that the third and fourth accent periods contain, not 8 beats, but 10 and 6 beats. Here, a listener should expect the last note to occur after 8 or 10 beats. But since the end actually occurs after 6 beats, the melody ends earlier than expected, and is predicted to be underestimated in relative duration. This should be particularly true for melodies ending on the leading tone interval where the end is now both tonally and temporally foreshortened in time. Conversely, this accent structure might attenuate overestimations that appeared in Experiment 1 when a tonic ending was judged relative to a leading-tone ending. The third type of manipulation is illustrated in Figure 1 with the melody labelled accent late. The third accent period of this tune contains 6 beats, leading a listener to expect the final note to occur after 8 or 6 beats. Since it actually occurs after 10 beats, the melody should appear to end "too late" in time and thus seem longer in relative duration. Overestimations may be further enhanced when a tonic ending is judged relative to a leadingtone ending since the melody appears to unfold along a more extended time course. Similarly, this particular accent structure may attenuate underestimations associated with a leading-tone ending.

In sum, the contrast model predicts systematic underand overestimations as a function of both tonal ending and temporal accent structure. These predictions, however, are counter to those generated by more traditional theories of time estimation. In terms of the storage size hypothesis, all melodies contain the same number of beats and the same number of chunks if we consider chunks to be marked by temporal accents. Therefore, all melo- 
dies should seem to have equivalent durations. Alternatively, it could be argued that melodies containing violations of rhythmic structure contain chunks of different lengths and are therefore more complex. Hence, these melodies might seem longer than the invariant tunes where there are no violations of rhythmic structure. The attentional effort models generate a similar prediction since rhythmic violations may enhance the degree of selective attention and thereby lengthen experienced duration. Lastly, the cognitive change model might claim that melodies with rhythmic violations contain more unpredictable changes in temporal structure and are therefore more complex. In sum, these three types of models predict that accent early and late melodies should either seem identical in total duration or longer than invariant melodies. Furthermore, if overestimations do arise, they should be significantly enhanced by the leading-tone ending. These predictions differ from those of the contrast model, which predicts both over- and underestimations as a function of rhythmic structure and tonal ending.

\section{Method}

Design and Subjects. The design was a $3 \times 2 \times 2 \times 2 \times 4$ $\times 2$ mixed factorial. Melodies within a pair always involved one of three folk tunes that could share a common (tonic-tonic; leading tone-leading tone) or different (tonic-leading tone; leading tone-tonic) tonal ending that varied in degree of resolution, and a common (invariant-invariant; accent early-accent early; accent late-accent late; invariant-invariant) or different (invariant-accent early; invariant-accent late; accent early-invariant; accent lateinvariant) temporal accent structure that varied in temporal disparity between actual and expected ending times. Two levels of counterbalance order (I, II) constituted the single between-subjects factor.

Twenty subjects from an introductory psychology course at Ohio State University participated in Experiment 2 in return for course credit. Each had had at least 4 years of musical experience within the past 6 years and was currently playing a musical instrument. Ten subjects were randomly assigned to each of the two counterbalance orders.

Stimulus materials. The songs from Experiment 1, along with their variations in tonal ending, were once again used as experimental stimuli. The temporal accent structure of each song, however, was varied to confirm or disconfirm expectancies about when the last note should occur. Recall that melodies in their original versions all contained agogic accents (i.e., $d$ ) that coincided with members of the tonic triad chord (i.e., tonic, mediant) where these coupled accents always recurred after an invariant number of beats (i.e., 8 beats). In the accent early condition shown in Figure 1, however, the temporal structure of the third and fourth accent periods was adjusted such that the number of beats were 10 and 6 , respectively, between coupled accents. Conversely, the temporal structure of the two final periods in the accent late condition was altered to contain 6 and 10 beats. Thus, relative to preceding accent periods, the final phrase ending within these two types of melodies occurred either "too early" or "too late" in time-despite the fact that all variants were equivalent in total duration and contained the same total numbers of beats.

Melodies were paired for presentation such that the temporal accent structure of each melody was either the same (invariant-invariant; accent early-accent early; accent late-accent late; invariant-invariant) or different (invariant-accent early; invariant-accent late; accent early-invariant; accent late-invariant). (The invariantinvariant condition was repeated a second time, such that an equal number of same and different accent structures would occur.) Similarly, the tonal ending for each melody in a pair could also be the same (tonic-tonic; leading tone-leading tone) or different (tonicleading tone; leading tone-tonic). Each melodic pair, however, always contained the same song and total duration. A factorial crossing of the three songs with the four conditions of tonal ending and eight conditions of temporal accent structure yielded a total of 96 experimental trials. These were randomized into two blocks of 48 trials.

Procedure and Apparatus. The subjects were randomly assigned to one of two counterbalance orders. During an experimental session, which was approximately $1 \mathrm{~h}$ long, each subject received a total of 96 trials. In all other respects, the apparatus and procedure were identical to those of Experiment 1.

\section{Results}

Table 2 depicts mean judged duration as a function of the tonal ending and temporal accent structure of the first and second melody within a pair. Means are collapsed over counterbalance order and song since both variables were nonsignificant.

Table 2

Mean Judged Duration in Experiment 2 (with Standard Deviations) as a Function of the Tonal Ending and Temporal Accent Structure of the First and Second Melody Within a Pair

\begin{tabular}{|c|c|c|c|c|c|c|c|c|c|}
\hline \multirow[b]{3}{*}{ Temporal Accent Pairs } & \multicolumn{8}{|c|}{ Tonal Ending Pairs } & \multirow[b]{3}{*}{ Mean } \\
\hline & \multicolumn{2}{|c|}{$\begin{array}{l}\text { Tonic- } \\
\text { Tonic }\end{array}$} & \multicolumn{2}{|c|}{$\begin{array}{l}\text { Leading- } \\
\text { Leading }\end{array}$} & \multicolumn{2}{|c|}{$\begin{array}{c}\text { Leading- } \\
\text { Tonic }\end{array}$} & \multicolumn{2}{|c|}{$\begin{array}{c}\text { Tonic- } \\
\text { Leading } \\
\end{array}$} & \\
\hline & $M$ & $S D$ & $M$ & $S D$ & $M$ & $S D$ & $M$ & $S D$ & \\
\hline Invariant-invariant & 4.00 & .21 & 4.00 & .14 & 4.50 & .33 & 3.71 & .27 & 4.05 \\
\hline Accent late-accent late & 4.00 & .29 & 4.00 & .25 & 4.54 & .34 & 3.73 & .31 & 4.07 \\
\hline Accent early-accent early & 4.00 & .27 & 4.00 & .42 & 4.51 & .35 & 3.68 & .31 & 4.05 \\
\hline Invariant-accent late & 4.78 & .66 & 4.69 & .43 & 5.10 & .51 & 3.92 & .60 & 4.62 \\
\hline Invariant-accent early & 3.22 & .34 & 3.30 & .40 & 4.13 & .37 & 2.94 & .38 & 3.40 \\
\hline Accent early-invariant & 4.51 & .47 & 4.53 & .45 & 4.80 & .47 & 4.02 & .45 & 4.47 \\
\hline Accent late-invariant & 3.48 & .32 & 3.55 & .40 & 4.05 & .42 & 3.19 & .35 & 3.57 \\
\hline Means & 4.00 & & 4.01 & & 4.52 & & 3.60 & & \\
\hline
\end{tabular}

Note-Invariant structures are those that presumably end "on time" while accent early and late structures are assumed to generate endings that occur "too early" and "too late" in time, respectively. A value of 4.00 indicates equivalent judged duration; values $<4.00$ indicate that the second melody was judged shorter; and values $>4.00$ indicate that the second melody was judged longer. 
As in Experiment 1, duration estimates were significantly influenced by melodic pairs that shared common or different tonal ending $\left[F(3,54)=13.58, M S_{\mathrm{e}}=0.154\right.$, $p<.001]$. Melodies that both ended on either the tonic or leading-tone interval were judged equivalent in total duration. However, underestimations of judged duration occurred when melodies ending on the leading-tone interval were paired with those ending on the tonic, while overestimations arose when the temporal order of this melodic pairing was reversed. When compared with the respective melodic pair containing the same tonal ending, Tukey post hoc tests confirmed that these differences were significant at $p<.01$.

Results also indicated a significant main effect for temporal accent structure $\left[F(6,108)=47.61, M S_{\mathrm{e}}=0.183\right.$, $p<.001$ ]. As illustrated in the row means of Table 2, melodies sharing a common accent structure were correctly judged the same in total duration. But relative to invariant accent structures that confirmed expectancies about when the last note should occur, melodies appearing to end "too early" (i.e., accent early) or "too late" (accent late) in time yielded systematic under- and overestimations, respectively (Tukey post hocs $<.01$.) Though not significant, there was also a slight temporal order effect; accent structures tended to exert a greater impact when the invariant pattern acted as the standard (vs. comparison) melody of a pair.

Although these effects of temporal accent structure generalized to melodies sharing the same tonal ending (i.e., tonic-tonic; leading tone-leading tone), a significant interaction $\left[F(18,324)=9.42, M S_{\mathrm{e}}=0.181\right.$, $p<.001]$ revealed that duration estimates of melodic pairs containing different tonal endings were selectively magnified or attenuated by temporal accent structure. Examining the third column of Table 2 , notice that tonic endings are overestimated relative to leading tone endings when both share the same accent structure $(p<.01$ for all Tukey post hoc comparisons). However, the degree of overestimation is significantly enhanced when the temporal accent structure of the second melody also leads to an ending that seems to occur relatively later in time (i.e., invariant-accent late and accent early-invariant; $p<$ $.01)$. Conversely, the degree of overestimation is markedly attenuated when the ending of comparison melodies seems to occur relatively earlier in time, (i.e., invariant-accent early and accent late-invariant; $p<.01$ ). The fourth column of Table 2 indicates that comparable findings emerge with melodic pairs containing tonic-leading-tone endings. Again the degree of underestimation observed for leading-tone endings is significantly enhanced when comparison patterns seem to end relatively too early $(p<.01)$, and reduced to judgments of equivalent duration when endings seem relatively late. In effect, then, the leading-tone interval no longer seems tonally foreshortened if temporal accent structure leads to an ending that is "too late" in time.

\section{GENERAL DISCUSSION}

The results of the present experiments lend support to a major assumption of the contrast model-namely that time estimates are influenced by the structural characteristics of the events to be judged. In the intepretation offered here, it is assumed that the predictability of event structure affords future-oriented attending and the generation of expectancies about how and when an event will end.

With respect to musical endings, there are at least two levels of structure that provide a basis for anticipatory attending. One stems from long-term exposure to music through which one has learned that most Western music ends on a progression from leading tone to tonic. Listeners rely on these pitch intervals to communicate musical completion. If a melody closes on the leading tone alone, it appears to be not only incomplete and to leave one "hanging" (Boltz, 1989, in press) but also short in relative duration. The second source of structurally based expectancies involves the melody's particular pattern of temporal accent structure. Given that temporal accents typically recur after a fixed number of beats and coincide with melodic phrase endings, a listener is able to use futureoriented attending and generate expectancies over higher order phrases. Together, tonal resolution and temporal accent structure provide the basis for attentional arcs that are targetted toward melodic endings. Tonal resolution specifies how a melody should end-namely on the interval from leading tone to tonic-and temporal accent structure specifies when in time this end will occur. And, as the present set of results indicates, the confirmation or violation of these joint expectancies exerts a primary influence on duration judgments. Melodies seem shorter if they end earlier than rhythmically expected, and the degree of underestimation markedly increases if they also appear tonally foreshortened in time by ending on the leading tone interval. In contrast, overestimations arise when the melody ends later than rhythmically expected, particularly if the melody also appears to unfold over a more extended tonal course by ending on the tonic interval. Overall, then, these results support predictions of the contrast model in that time estimates appear to be determined by the temporal disparity between expected and actual endings. They also converge with the results of Jones and Boltz (1989), whose manipulations of temporal contrast complement those achieved in the present study.

There is an alternative intepretation of the present findings that deserves mention. It is possible that effects of temporal accent structure were not mediated by expectancies, as assumed here, but by inferences based on the relative number of beats within a melody's final accent period. That is, even though subjects were instructed to judge the total duration of paired melodies, they may simply have relied on the heuristic of saying (e.g.) "longer" if the final accent period of the second melody contained a greater number of beats than that of the first melody. 
There are, however, two related findings suggesting that this was not the case. First, in a study by Boltz (1989), subjects were asked to judge the degree of completeness in melodies that varied in rhythmic accent structure. Results converged with the duration judgments observed here, in that the perceived resolution of tonal endings was highest when melodies ended "on time" through an invariant pattern of temporal accents. Significantly lower ratings were observed when accents led to endings occurring earlier or later than expected (i.e., accent early and late tunes). The second source of evidence suggesting the mediation of expectancies comes from a production study by Jones and Boltz (1989). Subjects were presented with tunes that varied in temporal accent structure and were asked to mentally extrapolate the duration of a melody's last three missing notes through a button press. Production times reflected listeners' expectancies, in that subjects showed overextrapolations for melodies containing a relatively fewer number of beats in their final accent periods (i.e., accent early tunes) and underextrapolations when final accent periods contained a relatively greater number of beats (i.e., accent late tunes). Melodies containing invariantly timed accents, however, yielded accurate production times that were nearly identical to the actual physical durations of the melodies' ends. Thus, these results suggest that subjects were using the melodies' overall rhythmic accent structures for anticipatory attending and that time-estimation activities were influenced by structurally based expectancies.

The overall pattern of results observed here presents difficulties for more traditional theories which assume that duration estimates depend on the amount of cognitive processing activity. From these perspectives, it is not clear why melodies ending on the leading tone interval are underestimated relative to those ending on the tonic. The tonic interval is the conventional marker of musical resolution, and it should therefore be less "complex" and require less processing activity. Similarly, the observed effects of temporal accent structure are also difficult to explain in terms of varying amounts of storage size, attentional effort, or cognitive change. The melodies of Experiment 2 were identical in all respects except for the relative placement of agogic accents. Thus, all the melodies should have appeared equivalent in total duration, or, alternatively, those with rhythmic violations should have seemed more complex and longer in relative duration. And yet this was not the case. Accent early melodies were judged shorter than accent late tunes, even though both displayed variations of rhythmic structure.

The present perspective suggests that these traditional models have difficulties because they fail to acknowledge the predictability of natural event structure and the function of preparatory attending that it affords. Although these models may be useful for explaining time judgments of less coherent events where people must "make sense" of seemingly unrelated items, corresponding applications to more predictable events are less successful. The present approach has suggested one means for reconciling these theoretical differences by proposing a continuum of structural coherence, along which time estimation activities vary as a function of the structural relationships within the events to be judged. Duration judgments of highly predictable events may depend on future-oriented attending and the generation of expectancies, whereas judgments of less predictable events may depend on the mediation of mnemonic and organizational strategies. In both cases, however, the common goal is to identify the nature of event structure and its impact on attending and perceiving activities.

\section{REFERENCES}

Allan, L. (1979). The perception of time. Perception \& Psychophysics, 26, 340-354.

Avant, L. L., \& LMan, P .J. (1975). Stimulus familiarity modifies perceived duration in prerecognition visual processing. Journal of $E x$ perimental Psychology: Human Perception \& Performance, 1, 205-211.

BERRY, W. (1976). Structural functions in music. Englewood Cliffs, NJ: Prentice Hall.

BLock, R. (1979). Time and consciousness. In G. Underwood \& R. Stevens (Eds.), Aspects of consciousness: Vol. 1. Psychological issues (pp. 179-178). London: Academic Press.

BLock, R. (1985). Contextual coding in memory: Studies of remembered duration. In J. A. Michon \& J. L. Jackson (Eds.), Time, mind, and behavior (pp. 169-178). Heidelberg: Springer Verlag.

BLock, R. (in press). Experiencing and remembering time: Affordances, context, and cognition. In I. Levin \& D. Zakay (Eds.), Time and human cognition: A life-span perspective. Amsterdam: North-Holland.

BLOCK, R., \& ReED, M. (1978). Remembered duration: Evidence for a contextual-change hypothesis. Journal of Experimental Psychology: Human Learning \& Memory, 4, 656-665.

Boltz, M. (1985). An expectancy model of judged duration: An ecological perspective. Unpublished doctoral dissertation, Ohio State University.

BoLtz, M. (1989). Rhythm and "good endings": Effects of temporal structure on tonality judgments. Perception \& Psychophysics, 46, 9-17.

Boltz, M. (in press). Perceiving the end: Effects of tonal relationships on melodic completion. Journal of Experimental Psychology: Human Perception \& Performance.

Browne, R. (1981). Tonal implications of the diatonic set. In Theory Only, 5, 3-21.

BuRNSIDE, W. (1971). Judgment of short time intervals while performing mathematical tasks. Perception \& Psychophysics, 9, 404-406.

BuTler, D., \& Brown, H. (1984). Tonal structure versus function: Studies of the recognition of harmonic motion. Music Perception, 2, 6-24.

CoNE, E. (1968). Musical form and musical performance. New York: W. W. Norton.

COOPER, W. E., PACcia, J. M., LAPointe, S. G. (1978). Hierarchical coding in speech timing. Cognitive Psychology, 10, 154-177.

CoOPER, W. E., \& Sorensen, J. M. (1977). Fundamental frequency contours at syntactic boundaries. Journal of the Acoustical Society of America, 62, 683-692.

Cuddy, L., Cohen, A., \& Miller, J. (1979). Melody recognition: The experimental application of musical rules. Canadian Journal of Psychology, 33, 148-157.

DeVANE, J. (1974). Word characteristics and judged duration for two response sequences. Perceptual \& Motor Skills, 38, 525-526.

Fraisse, P. (1984). Perception and estimation of time. Annual Review of Psychology, 35, 1-36.

HoGaN, H. (1975). Time perception and stimulus preference as a function of stimulus complexity. Journal of Personality \& Social Psychology, 31, 32-35. 
JoNES, M. R. (1976). Time, our lost dimension: Toward a new theory of perception, attention, and memory. Psychological Review, 83, 323-335.

JONES, M. R. (1982). Music as a stimulus for psychological motion: Part II. An expectancy model. Psychomusicology, 2, 1-13.

JoNES, M. R. \& BoLTZ, M. (1989). Dynamic attending and responses to time. Psychological Review, 96, 459-491.

Jones, M. R., BolTz, M., KIDD, G. (1982). Controlled attending as a function of melodic and temporal context. Perception \& Psychophysics, 32, 211-218.

Kramer, J. (1981). New temporalities in music. Critical Inquiry, 7, 539-556.

Meyer, L. (1956). Emotion and meaning in music. Chicago: University of Chicago Press.

ORNSTEIn, R. (1969). On the experience of time. Baltimore: Penguin. Piston, W. (1978). Harmony (4th ed., rev. and exp. by M. DeVoto). New York: W. W. Norton.

PoYNTER, W. D. (1983). Duration judgment and the segmentation of experience. Memory \& Cognition, 11, 77-82.
SCHIFFMAN, H., \& BoBKo, D. (1974). Effects of stimulus complexity on the perception of brief temporal intervals. Journal of Experimental Psychology, 103, 156-159.

ScotT, D. (1982). Duration as a cue to the perception of a phrase boundary. Journal of the Acoustical Society of America, 71, 996-1007.

Sundberg, J., FrIberg, A., \& Frydén, L. (1987). How to terminate a phrase: An analysis-by-synthesis experiment on perceptual aspects of music performance. In A. Gabrielsson (Ed.), Action and perception in rhythm and music (pp. 49-55). Stockholm: The Royal Swedish Academy of Music Publications.

Thomas, E. A. C., Weaver, W. B. (1975). Cognitive processing and time perception. Perception \& Psychophysics, 17, 363-367.

Todv, N. (1985). A model of expressive timing in tonal music. Music Perception, 3, 33-58.

UNDERWOOD, G. , SWAIN, R. (1973). Selectivity of attention and the perception of duration. Perception, 2, 101-105.

(Manuscript received August 15, 1988; revision accepted for publication March 28, 1989.) 